\title{
CDPath: Cooperative driver pathways discovery using integer linear programming and Markov clustering
}

\author{
Ziying Yang, Guoxian Yu, Maozu Guo, Jiantao Yu, Xiangliang Zhang and Jun Wang
}

\begin{abstract}
Discovering driver pathways is an essential task to understand the pathogenesis of cancer and to design precise treatments for cancer patients. Increasing evidences have been indicating that multiple pathways often function cooperatively in carcinogenesis. In this study, we propose an approach called CDPath to discover cooperative driver pathways. CDPath firstly uses Integer Linear Programming to explore driver core modules from mutation profiles by enforcing cooccurrence and functional interaction relations between modules, and by maximizing the mutual exclusivity and coverage within modules. Next, to enforce cooperation of pathways and help the follow-up exact cooperative driver pathways discovery, it performs Markov clustering on pathway-pathway interaction network to cluster pathways. After that, it identifies pathways in different modules but in the same clusters as cooperative driver pathways. We apply CDPath on two TCGA datasets: breast cancer (BRCA) and endometrial cancer (UCEC). The results show that CDPath can identify known (i.e., TP53) and potential driver genes (i.e., SPTBN2). In addition, the identified cooperative driver pathways are related with the target cancer, and they are involved with carcinogenesis and several key biological processes. CDPath can uncover more potential biological associations between pathways (over 100\%) and more cooperative driver pathways (over $200 \%$ ) than competitive approaches. The demo codes of CDPath are available at http://mlda.swu.edu.cn/codes.php?name=CDPath
\end{abstract}

Index Terms-Cooperative Driver Pathway, Data Fusion, Integer Linear Programming, Markov Clustering.

\section{INTRODUCTION}

C Ancer is a disease with high mortality rate and driven in part by somatic mutations, which accumulate during the lifetime of an individual. With the reducing costs of genome sequencing, projects (i.e., The Cancer Genome Atlas (TCGA) |1] and International Cancer Genome Consortium (ICGC) [2]) have been initiated to undertake the task of measuring these somatic mutations in hundreds of samples from dozens of cancer types. A key challenge in interpreting these data is to distinguish the functional driver mutations,

Z. Yang, G. Yu and J. Wang are with the College of Computer and Information Sciences, Southwest University, Chongqing, China.

M. Guo is with the School of Electrical and Information Engineering, Beijing University of Civil Engineering and Architecture, Beijing, China.

$J . \mathrm{Yu}$ is with the College of Information Engineering, North-West AEF University, Shaanxi, China

$X$. Zhang is with the King Abdullah University of Science and Technology, Thuwal, $S A$

M. Guo and J. Wang are the corresponding authors. Email: guomaozu@bucea.edu.cn (M. Guo), kingjun@swu.edu.cn (J. Wang).

Manuscript received 12 May, 2019; Revised $x x \times x x$, xxxx which are important to cancer development from random passenger mutations that have no consequence for cancer, these driver mutations contribute to improve the diagnosis and treatment of cancer patients.

Whether a mutation is a driver or a passenger can be tested by its biological function. However, the capability of detecting somatic mutations currently far exceeds the capability of experimentally validating their functions. Therefore, effective computational approaches for large scale driver mutation detection are urgently needed. Most early efforts devoted to the detection of individual driver genes with significantly higher mutation rate [3]. For example, Ding et al. [4] used synonymous somatic mutations identified in 250 genes to estimate the background mutation rate, and then identified 26 genes significantly mutating in 188 human lung adenocarcinomas. However, this kind of methods unreasonably consider the rate as a constant value for all samples in the entire genome, and therefore ignore the heterogeneity of genome aberrations [5].

Different gene mutations may target at the same pathway. In recent years, more attention have been paid to identify driver pathways and modules rather than individual genes [6], |7], these pathways and modules can capture the heterogeneous pattern in cancer. Many driver pathway identification methods involve the use of prior knowledge, like pathways and/or protein interaction networks. For example, HotNet [8] uses a thermal diffusion process on interactive networks to identify individual driver pathways. On the other hand, several studies indicate that mutations in driver pathways often cover a large number of samples (i.e. high coverage) and usually exhibit mutual exclusivity [9]. These characteristics have been frequently used to detect driver pathways and modules [10]. For example, Ciriello et al. |11| proposed MEMo (Mutual Exclusivity Modules) to detect driver modules within a reconstructed network using gene mutation information and prior knowledge (including protein interactions and signal transduction pathways). However, the prior knowledge of raw protein interactions is usually incomplete and noisy, and a large portion of knowledge about biological pathways structure (mainly gene-pathway association) remains unclear, they both may reduce the reliability of experimental results. Furthermore, using which kind of prior knowledge is dependent on the researcher's priority and background. 
To avoid these limitations of prior knowledge based methods [8], [11], researchers develop new methods to discover mutated driver pathways or modules without relying on prior information. Vandin et al. [12] proposed Dendrix (de novo driver exclusivity) to de novo discover mutated driver pathways using somatic mutation data. Dendrix introduces a novel weight function to search genes, which mutate with high coverage and mutual exclusivity, and thus to extract mutual exclusively mutated gene set. Maximization of the weight function is defined as the maximum weight submatrix problem, which is originally solved by Markov Chain Monte Carlo (MCMC) method [12], and then addressed by an exact binary linear programming (BLP) model [13|. Zhang et al. |14| proposed two optimization models to de novo separately discover common driver gene sets among multiple cancer types (ComMDP) and specific driver gene sets of a particular cancer (SpeMDP). However, these methods focus on single pathways or modules [12]-[14], they can not clarify how various cellular and physiological processes are coordinately altered during the initiation and progression of cancer.

It has been recognized that pathways often function cooperatively in carcinogenesis [15]. Mutations of two genes participating in the same pathway or process rarely confer a significant selective advantage compared to the single mutation, since the functional consequences of single and double mutations are similar. For example, gene pair (EGFR, KRAS) is mutated with a coverage overlap 0 in lung adenocarcinoma patients, indicating mutual exclusivity [12]. In contrast, functional consequences of mutations of multiple genes that participate in different pathways or functions may be additive or even synergistic in conferring an advantage to the tumor. Therefore, we would expect to observe a tendency of mutually exclusive mutations of genes in the same pathway (mutual exclusivity) and the tendency of co-occurring mutations of genes that compose distinct pathways (co-occurrence) [16]. By identifying oncogenesignaling blocks from integrated human signaling network, Cui et al. $\mid 17$ found that some blocks (such as the RAS and TP53) collaboratively promote cancer signaling and tumorigenesis. $\mathrm{Gu}$ et al. $|18|$ uncovered pathway cooperation in cancer cells in significantly co-disrupted pathways using 18 pathways enriched with mutations in lung adenocarcinoma. Gu et al. [19] further identified cooperative functional modules in the glioblastoma multiforme (GBM) altered network by mapping mutated genes onto a protein interaction network.

All these studies $[16]-[19]$ indicate that the malignant transformation from a normal cell to a tumor is involved with synergy cooperations between pathways. Therefore, systematically exploring the complex collaboration among multiple biological pathways and functional modules is a crucial step to improve our understanding of the cellular mechanisms underlying tumorigenesis. However, these aforementioned methods rely on the utilization of prior knowledge (gene-pathway association) to determine whether multiple pathways or modules are significantly perturbed. Considering the incomplete prior knowledge about pathways structure and protein interaction networks, researchers introduce de novo approaches to explore cooperative driver pathways based on mutation data. CoMDP $[20 \mid$ builds a mathematical programming model to maximize an extended weight function [12] and to find pairwise cooperative driver pathways. Leiserson et al. [21] proposed Multi-Dendrix to optimize the weight function, which uses a sum of multiple quantities for multiple pathways, to identify multiple driver pathways. These de novo cooperative pathways identification methods still have limitations. They consider only mutation data, and thus may miss important information (like pathway and gene interactions) in carcinogenesis, and may be easily trapped in suboptimal solution. In addition, these methods have to specify the number of driver genes in advance, and may produce biased results.

In this study, we introduce a computational approach called CDPath that integrates mutation data and prior knowledge to discover cooperative driver pathways. Firstly, CDPath identifies driver core modules by simultaneously maximizing the coverage and mutual exclusivity within modules, and the co-occurrence and functional interactions between modules. CDPath formulates the module identification problem as an Integer Linear Programming (ILP) problem, which can avoid presetting the number of driver genes [22] and incorporate constraints to guide the identification. Next, to obtain more reliable cooperation between pathways and to help the follow-up exact cooperative driver pathways discovery, CDPath uses Markov clustering (MCL) [23] on pathway interaction (PaPaI) network to group pathways with functional relations and to avoid losing cooperative driver pathways caused by incomplete genepathway association information. After that, CDPath takes the pathways in different modules but in the same clusters as cooperative driver pathways.

We apply CDPath on two TCGA [1] datasets: somatic mutation profiles in breast cancer (BRCA) and endometrial cancer (UCEC). The experimental results show that CDPath can identify known driver genes. After GO and KEGG enrichment analysis, more than $90 \%$ genes in the core modules show strong relations with cancer pathways and with important biological activities. The identified cooperative driver pathways are also related with the target cancer and other cancers. In summary, CDPath can discover more potential biological associations between genes and pathways than previous methods that can only identify individual driver pathways [12], [13], and it can also discover more cooperative driver pathways than existing competitive methods [20], [21].

\section{Methods}

CDPath follows the procedure described in Figure 1 to discover cooperative driver pathways. Firstly, it solves the driver core module identification problem by ILP and gets cooperative driver modules. Next, it uses MCL on the PaPaI network to get pathway clusters that group pathways with functional cooperations. After that, it combines the results of driver core modules and pathway clusters, and uses a coverage based method to identify cooperative driver pathways, which are in different driver core modules but in the same pathway clusters. The following subsections will elaborate on these procedures. 
Modules Identified by ILP

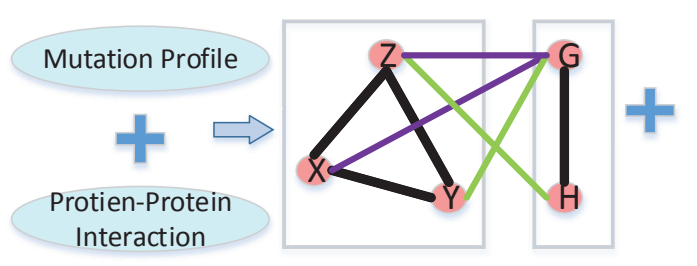

Gene-Pathway Association

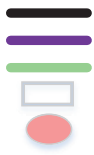

Mutually Exclusive Mutation Functional Interaction Co-occurring Mutation Module Gene

\section{Pathway Modules Clusters Identified by MCL}

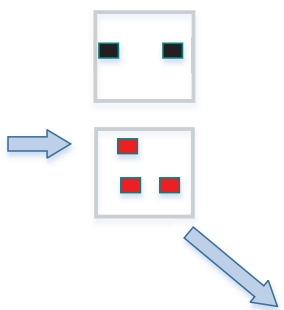

Pathway Interaction Network



Driver Pathway Identification Fig. 1. The workflow of CDPath. CDPath firstly uses the Integer Linear Programming (ILP) to maximize the mutual exclusivity, coverage, functional
interaction and co-occurrence to find cooperative driver core modules. By using known gene-pathway association data, we can get driver pathway modules. Next, CDPath performs Markov clustering (MCL) on the pathway interaction network to get interactive pathway clusters. CDPath finally utilizes the driver pathway modules and pathway clusters to identify the pathway sets belonging to different modules but in the same cluster as cooperative driver pathways.

\subsection{Cooperative driver modules identification}

Beside the characteristic of high coverage and mutual exclusivity in the same pathway and of large co-occurrence between multiple pathways, we further make use of functional interactions between genes as prior knowledge, which helps uncovering the cooperation between pathways at the gene level. Specifically, we split the interactions with low confidence to decrease the interference of noisy interactions and to mitigate the limitation of incomplete prior knowledge of gene interactions. We transform the cooperative driver pathway identification problem into a driver core module identification problem. Particularly, given a set of genes and two types of edge scoring functions (within and between scores), we aim to find modules that genes within a module have the minimum 'within' scores (mutual exclusivity and coverage), while gene pairs spanning two different modules have the maximum 'between' scores (cooccurrence and functional interactions). We formulate this module identification problem as an ILP problem and detail it as follows.

Let $N_{m}$ be the number of identified modules, $M_{g}$ be the maximum number of genes per module, and $\mathcal{N}_{g}$ be the set of candidate genes with mutations. We aim to divide genes into $N_{m}+1$ modules, where the $\left(N_{m}+1\right)$-th module includes all the unselected genes. We use the binary variable $y_{i k}$ to indicate whether gene $i$ is in module $k\left(y_{i k}=1\right)$, or $y_{i k}=0$ otherwise. In addition, the following integer binary variables are used to indicate different types of gene pairs: $w_{i j k}=1$ if gene $i$ and $j$ are in the same module $k, 0$ otherwise. $b_{i j}=1$ if gene $i$ and $j$ are in the different modules, 0 otherwise. $u_{i j}=1$ if both gene $i$ and $j$ are unselected, 0 otherwise. Then the objective of ILP is defined as:

$$
\operatorname{Max} \sum_{i, j \in \mathcal{N}_{g}} \operatorname{between}(i, j) b_{i j}+\sum_{i, j \in \mathcal{N}_{g}} \sum_{k=1}^{N_{m}} \operatorname{within}(i, j) w_{i j k}
$$

where between $(i, j)$ and within $(i, j)$ are two weight functions for pairs of genes between and within modules, re- spectively. between $(i, j)=w_{C O}(i j)+w_{F}(i j)-w_{M E}(i j)$ is utilized to reward co-occurrence and functional interactions, and to penalize mutual exclusivity between modules. within $(i, j)=\operatorname{cov}(i j)+w_{M E}(i j)-w_{C O}(i j)-w_{F}(i j)$ is used to enforce mutual exclusivity and reward coverage, and to penalize co-occurring mutations and functional interactions within modules. $\operatorname{cov}(i j)$ is the union coverage of gene $i$ and gene $j$. Let $\mathcal{S}_{i}$ be the sample set that gene $i$ mutates in, $\operatorname{cov}(i j)$ is proportional to $\left|\mathcal{S}_{i} \cup \mathcal{S}_{j}\right|$, which ensures $\operatorname{cov}(i j)$ increasing along with the increase of mutations of gene $i$ and $j$. The weight $w_{F}(i j)$ is to quantify the functional relations. It is defined based on the confidence scores of respective protein-protein interactions. The weight $w_{M E}(i j)$ and $w_{C O}(i j)$ are introduced to quantify the mutual exclusivity and co-occurrence for each pair of mutations, they are defined based on $p$-values obtained by weighted sampling based methods (WeSME and WeSCO [24]), which can test mutual exclusivity and co-occurrence, respectively. $w_{M E}(i j)$ and $w_{C O}(i j)$ are set to 0 if there is no edge between gene $i$ and $j$.

Eq. (1) can then be reformulated as:

$$
\begin{aligned}
& \operatorname{Max} \sum_{i, j \in \mathcal{N}_{g}}\left(w_{C O}(i j)-w_{M E}(i j)+w_{F}(i j)\right) b_{i j} \\
& +\sum_{i, j \in \mathcal{N}_{g}} \sum_{k=1}^{N_{m}}\left(\operatorname{cov}(i j)+w_{M E}(i j)-w_{C O}(i j)-w_{F}(i j)\right) w_{i j k}
\end{aligned}
$$

To get the optimal and feasible solution, we incorporate some constraints into Eq. (2). The set of constraints defined in Eq. (3) ensure that $w_{i j k}=1$ if both $i$ and $j$ are placed into the $k$-th module.

$$
\begin{array}{ll}
w_{i j k} \leq y_{i k} & \forall i j, \forall k \in\left[1, N_{m}\right] \\
w_{i j k} \leq y_{j k} & \forall i j, \forall k \in\left[1, N_{m}\right] \\
w_{i j k} \geq y_{i k}+y_{j k}-1 & \forall i j, \forall k \in\left[1, N_{m}\right]
\end{array}
$$

Similarly, for each gene pair $i$ and $j$, they are either unselected, between modules, or in the same module. As 
such, they need to satisfy the set of constraints formulated in Eq. (4) to ensure the proper assignment of $u_{i j}$ and $b_{i j}$.

$$
\begin{array}{ll}
u_{i j} \geq y_{i N_{m}^{\prime}} & \forall i j \\
u_{i j} \geq y_{j N_{m}^{\prime}} & \forall i j \\
u_{i j} \leq y_{i N_{m}^{\prime}}+y_{j N_{m}^{\prime}} & \forall i j \\
b_{i j}=1-u_{i j}-\sum_{k=1}^{N_{m}} w_{i j k} & \forall i j
\end{array}
$$

To avoid the main effect of a super significant driver gene (like TP53, each gene with it will be significant), which mutates in the majority of samples (over $80 \%$ ), we additionally include the constraints in Eq. (5) to ensure that each gene $i$ belongs to exact one module, and to bound the module size by $M_{g}$ to avoid too much genes in a module and identifying no significant modules.

$$
\begin{array}{ll}
\sum_{k=1}^{N_{m}+1} y_{i k}=1 & \forall i \in \mathcal{N}_{g} \\
\sum_{i \in \mathcal{N}_{g}} y_{i k} \leq M_{g} & \forall k \in\left[1, N_{m}\right]
\end{array}
$$

To make genes within modules being in the same pathways and playing important roles in the PPI network, we force each module including a dense subnetwork of the protein functional interaction network using constraints in Eq. (6):

$$
\begin{aligned}
& \sum_{j \in\left\{j^{\prime} \mid \exists i j^{\prime} \in \mathcal{E}_{F U}\right\}} y_{j k} \geq D\left(M_{g}-1\right)\left(y_{i k}-1\right) \\
& +D\left(\sum_{j \in \mathcal{N}_{g}} y_{j k}-1\right) \quad \forall i \in \mathcal{N}_{g}, \forall k \in\left[1, N_{m}\right]
\end{aligned}
$$

where $\mathcal{E}_{F U}$ is a subset of gene pairs with significant functional interactions. The constraints ensure that each gene $i$ in module $k$ is connected with at least $D$ fraction of genes in module $k$ via functional edges of $\mathcal{E}_{F U}$.

In order to finally identify cooperative driver pathways, we strengthen cooperation between modules by additionally requiring that for each module, it has at least one cooccurrence edge with other modules:

$$
\sum_{j \in\left\{j^{\prime} \mid i j^{\prime} \in \mathcal{E}_{C O}\right\}} b_{i j} \geq y_{i k} \quad \forall i \in \mathcal{N}_{g}, \forall k \in\left[1, N_{m}\right]
$$

where $\mathcal{E}_{C O}$ is a subset of gene pairs with significant cooccurrence.

Although variables $w_{i j k}, u_{i j}, b_{i j}$ are required to be binary, we feasibly relax them as continuous in $[0,1]$. In this way, all the target variables in the optimal solution are still binary but the running time can be significantly reduced. Our preliminary study in Section 1 and Table S1 of the Supplementary file confirms this advantage. To further improve the efficiency, we apply a symmetry breaking technique, which allows for equivalent solutions to speed up the calculation of ILP. In addition, by adding constraints on the feasible solution set (e.g., only acquiring for 3 types of edges), the sharply increased running time and memory usage caused by brand-and-bound trees of symmetry breaking technique, can be greatly alleviated. After applying ILP on the mutation data, we can identify the driver core modules, which will be used to identify exact cooperative driver pathways in the following procedures.

\subsection{Clustering on pathway interaction network}

Although ILP already identifies the driver core modules, the associations between genes and pathways are not complete enough to correspond these gene modules to pathways. For a gene in the identified modules, several related pathways can be found but these pathways may have low coverage, which makes it hard to directly correspond modules with pathways. To get exact cooperative driver pathways, for each gene in a module, we collect the related pathways together as pathway module. Then, we only need to find pathways with cooperation, which belong to different pathway modules, as cooperative driver pathways. For this purpose, we further enforce functional interaction between pathways at pathway level by Markov clustering (MCL) [23] on the $\mathrm{PaPaI}$ network. Markov clustering have been emerging as an effective algorithm for clustering biological networks and widely used to identify functional modules [25]-[27]. After clustering, pathways with close functional relations will be grouped into the same clusters, which will be used for follow-up exact cooperative pathway identification.

Let $G=\left(\mathcal{N}_{p}, \mathcal{E}_{p}\right)$ be the input undirected graph of the collected PaPaI network, where $\mathcal{N}_{p}$ denotes the node set and $\mathcal{E}_{p}$ stands for the edge set. Let $p_{i} \in \mathcal{N}_{p}$ be the $i$-th pathway, $w\left(p_{i}, p_{j}\right)$ is the weight of edge $\left(p_{i}, p_{j}\right), w\left(p_{i}, p_{j}\right)=1$ if there is an interaction between $p_{i}$ and $p_{j}$ in the PaPaI network, 0 otherwise. $\mathbf{P} \in \mathbb{R}^{\left|\mathcal{N}_{p}\right| \times\left|\mathcal{N}_{p}\right|}$ is the adjacency matrix of $G, \mathbf{P}$ is initialized as follows:

$$
\mathbf{P}(i, j)=\left\{\begin{array}{cc}
w\left(p_{i}, p_{j}\right) & \text { if }\left(p_{i}, p_{j}\right) \in \mathcal{E}_{p} \\
\max _{k \neq j} w\left(p_{i}, p_{k}\right) & \text { if }\left(p_{i}=p_{j}\right), k \in\left[1,\left|\mathcal{N}_{p}\right|\right] \\
0 & \text { otherwise }
\end{array}\right.
$$

A canonical flow matrix $\mathbf{P}$ stores the probabilities of transition of a Markov chain defined on the graph. $\mathbf{P}(i, j)$ represents the probability of a transition from $p_{i}$ to $p_{j}$. Specifically, we use the maximum weight of edge in $\mathrm{PaPaI}$ to indicate $w\left(p_{i}, p_{j}\right)$ if $i=j$. We also refer to the transition probability from $p_{i}$ to $p_{j}$ as the stochastic flow or simply the flow from $p_{i}$ to $p_{j}$. Note that all elements in each column of $\mathbf{P}$ sum up to 1 . The matrix is normalized as:

$$
\tilde{\mathbf{P}}(i, j)=\frac{\mathbf{P}(i, j)}{\sum_{k=1}^{\left|\mathcal{N}_{p}\right|} \mathbf{P}(k, j)}
$$

The MCL process consists of two operations, namely 'Expand' and 'Inflate'. MCL iteratively assigns the 'Expand' and 'Inflate' matrix to the stochastic matrix, respectively. The Expand operation is simply expressed as:

$$
\mathbf{P}_{e x p}=\tilde{\mathbf{P}} * \tilde{\mathbf{P}}
$$

The Inflate operation raises each entry in the matrix $\tilde{\mathbf{P}}$ using the inflation parameter $r p(r p>1$, default 2$)$ followed by re-normalizing the sum of elements in each column to 1 . The Inflate operation can exaggerate the inhomogeneity in each column. In other words, flow is strengthened where it is already strong and weakened where it is weak. This Inflate operation is formulated as Eq. (11) as follows:

$$
\mathbf{P}_{\text {inf }}(i, j)=\frac{\tilde{\mathbf{P}}(i, j)^{r p}}{\sum_{k=1}^{\left|\mathcal{N}_{p}\right|} \tilde{\mathbf{P}}(k, j)^{r p}}
$$

MCL starts with the canonical flow matrix $\mathbf{P}$ and iteratively applies the above Expand and Inflate operations until 
convergency. After convergency, each column has only one non-zero entry in the final flow matrix, and these non-zero entries in the same row form a cluster of pathway nodes. After that, we can identify interactive pathway clusters by leveraging the driver core modules and pathway clusters.

\subsection{Cooperative driver pathway identification}

Cooperative driver pathways should have: (i) high mutual exclusivity and coverage within a pathway; (ii) high cooccurrence between pathways; (iii) large functional cooperation between pathways. There are tight associations between genes and pathways, based on the discovered cooperative driver core modules and the interactive pathway clusters, so we can identify exact cooperative driver pathways by combining these modules and pathways. To reach this goal, we propose a coverage based method and identify pathways that belong to different modules and the same clusters as cooperative driver pathways.

Based on gene-pathway association information, we can get a set of pathways related with these modules. We then can divide these pathways into pathway modules based on common genes with each pathway. Suppose that there are $N_{c}$ pathway clusters and $N_{m}$ pathway modules, for the $i$ th pathway cluster $\mathcal{C}_{p}^{i}$, we can identify its corresponding cooperative driver pathway sets $\mathcal{P}_{c o}^{i}$ as follows:

$$
\mathcal{P}_{c o}^{i}=\left\{\mathcal{C}_{p}^{i} \cap \mathcal{M}_{p}^{j} \mid j=1,2, \cdots, N_{m}\right\}
$$

where $\mathcal{M}_{p}^{j}$ is the $j$-th pathway module. We enumerate cooperative driver pathway sets from each intersection of gene modules and a pathway cluster, and then identify the intersection, whose related genes belong to different modules as the cooperative driver pathways. By iteratively identifying the corresponding cooperative driver pathway sets for $N_{c}$ pathway clusters, we can finally get the cooperative driver pathways.

\section{EXPERIMENT RESULTS AND ANALYSIS}

\subsection{Data source and processing}

We apply CDPath on two cancer datasets collected from TCGA [1]: somatic mutation profiles of 665 BRCA samples in breast cancer (BRCA), 207 UCEC samples in endometrial cancer (UCEC) (version 20180110). The protein-protein interaction network is obtained from Dao et al. [22] (version $10.4)$, and the interactions with low confidence scores $(<900)$ are split to decrease the noise. The PaPaI network data and gene-pathway association data are downloaded from PID databse [28] (version 20171020), and composed with 212 pathway nodes.

The maximal number of genes in a driver core module $M_{g}$ is set as a default value 10 , same as the setting in |22|. That is because a module containing too much genes is extremely hard to mutate with mutual exclusivity, while too few genes makes it hard to ensure the high coverage. In fact, previous work [13], [20] usually set the number of genes in a pathway no more than 10 genes. We test CDPath with different densities of modules $D$ and report the result in Section 2 of the supplementary file. We observe that $D$ has little influence on the objective function value and the number of identified driver genes. Therefore, we set $D$ as 0.7 with the maximum objective function value. As for the number of genes $N_{m}$, we set $N_{m}=8$ and $N_{m}=6$ for BRCA and UCEC dataset with the maximal driver genes, respectively. The detailed parameter analysis is also provided in Section 2 of the supplementary file for space limit.

\subsection{CDPath on stimulation data}

To validate the rationality and effectiveness of CDPath, we compute the significance of the results compared to 10 randomized instances (with different types of randomized edges). We evaluate the modules obtained in each randomization based on the proposed ILP function and constraints, and how well the modules identify known driver genes. For the list of cancer drivers, we use a combined list from COSMIC Cancer Gene Census [29]. To show how well the proposed CDPath can identify modules with desired characteristics in the presence of noise, we generate noisy simulated data, and then apply CDPath on the noisy data and evaluate its robustness based on the accuracy of identifying correct modules.

\subsubsection{CDPath on random instances}

For each randomized instance, we first randomize the protein-protein interactions by swapping interactions to preserve node degree distribution. For interaction swapping, we randomly choose two interactions without common proteins and swap their proteins. As a result, two new interactions are created. We do the swap change only for the nodes of the randomly selected edges. As for other unselected edges of the nodes, we do not change them. We repeat the interaction swapping process at least 100 times as suggested [22]. To randomize ME/CO edges, we randomly sample the $p$-value for the gene pairs from BRCA data with WeSME/WeSCO $p$-value smaller than 0.25 to ensure that the $p$-values of random instances close to real data. Similarly, to randomize coverage of genes, we sample the coverage from real BRCA data. In this way, 50 randomized instances are generated.

As Table 1 shows, when we randomize any kind of edges (ME, CO, functional edges and coverage), the number of identified driver genes, and the objective function score slightly decrease on functional edges randomization, but drops sharply on other kinds of randomization. This phenomenon shows that each kind of edges are important to identify cancer modules, especially $\mathrm{ME}, \mathrm{CO}$ edges and coverage. The reason for the slight decrease under the randomization of functional edges is that functional edges are much less than other edges in the identified modules. We can see that even with different edges randomization, the driver genes enrichments are still significant, this observation further confirms the effectiveness of the adopted ILP-based objective function in identifying cooperative core modules.

\subsubsection{CDPath on noisy data}

In this subsection, we follow the procedure used in et al. [22] to generate simulated data with noises, and then test the robustness of CDPath on these simulated data. Particularly, we firstly create a random functional network by randomly 
TABLE 1

Comparison of the results of CDPath on real and randomized BRCA data.

\begin{tabular}{l|l|l|l}
\hline Features & Drivers & Drivers Enrichment & Value \\
\hline Real & $\mathbf{1 1}$ & $\mathbf{6 . 6 6 E - 1 6}$ & $\mathbf{1 3 8 . 5 6}$ \\
\hline All edges & 4.6 & $1.60 \mathrm{E}-06$ & 22.29 \\
\hline Functional edges & 8.9 & $1.93 \mathrm{E}-11$ & 128.70 \\
\hline ME edges & 4.3 & $1.07 \mathrm{E}-06$ & 22.41 \\
\hline CO edges & 6.8 & $7.65 \mathrm{E}-08$ & 78.45 \\
\hline Coverage & 8.6 & $1.37 \mathrm{E}-11$ & 74.35 \\
\hline
\end{tabular}

Features: different kinds of randomization. Drivers: average number of driver genes in the identified modules. Driver Enrichment: driver genes enrichment calculated by hypergeometric test. Value: average objective function value. The number of modules is set the same as BRCA dataset.

choosing a subnetwork from protein-protein network of 1000 genes and swapping the functional interaction edges of the subnetwork (as mentioned before). This swapped subnetwork will be used to provide functional edges. Next, we generate the $\mathrm{ME}$, coverage and $\mathrm{CO}$ edges of simulated data. Let $P_{M E}$ and $P_{C O}$ be the list of $p$-values from WeSME and WeSCO tests, and $P_{\text {cov }}$ be the union coverage list on BRCA dataset. For each gene pair, we randomly sample a $p$-value from the list of $P_{M E}$ and add an ME edge if $p \leq 0.01$, which ensures having a larger weight than the STRING functional edges. The $\mathrm{CO}$ edges and coverage are also similarly added. We then randomly generate $k(2 \leq k \leq 4)$ modules in the generated network, each of which is composed with 2-5 genes and each gene belongs to only one module. In order to make sure that these modules satisfy the desired characteristics, for each gene $g$ in a module $M$, we randomly choose $80 \%$ of genes in other modules and additionally add functional (sampled from protein-protein network) and $\mathrm{CO}$ edges (sampled from $P_{C O}$ with $p$-value smaller than 0.01 ) between $g$ and these genes. Similarly, for each gene $g$ in a module $M$, we randomly choose $80 \%$ of other member genes in $M$, and add ME edges (sampled from $P_{M E}$ with $p$-value smaller than 0.01) and union coverage (randomly sampled from $P_{\text {cov }}(>100)$ ). When we add an $\mathrm{ME} / \mathrm{CO} /$ functional/coverage edge between a pair of genes, we also remove an existing corresponding edge. Finally, with a probability $r_{n}$, we randomly add noise by replacing the $p$-value of the $\operatorname{ME}(\mathrm{CO})$ edge with a random $p$-value $>0.01$ sampled from $P_{M E}$ and $P_{C O}$.

We apply CDPath on 10 simulated datasets with noises injected as stated above and set the within density $D=0.6$. We use different noise levels $r_{n}(0.1,0.2$ and 0.3$)$ and evaluate the accuracy of our method. For each noise level, we report the accuracy (the fraction of correctly identified modules among 10 simulated datasets) and partial match (over $75 \%$ genes in the modules are identified) of our method. As Table 2 shows, with the increase of the noise level, accuracy drops but the partial match remains high, which demonstrates the robustness of our method. Specially, when there is no noise, CDPath can always perfectly identify all the correct modules. These experiments suggest that CDPath can effectively identify modules with high coverage and mutual exclusivity within modules, and with high co-occurrence and functional relations between modules, which are important characteristics of cooperative driver
TABLE 2

The robustness of CDPath with simulated data.

\begin{tabular}{l|l|l}
\hline Noise Level $r_{n}$ & Accuracy & Partial Match Percentage \\
\hline 0 & 0.99 & 1 \\
\hline 0.1 & 0.93 & 1 \\
\hline 0.2 & 0.85 & 0.99 \\
\hline 0.3 & 0.74 & 0.97 \\
\hline
\end{tabular}

pathways.

\subsection{CDPath on real biological data}

\subsubsection{Identified driver modules analysis}

As Table 3 and Table 4 show, with different settings of $N_{m}$ (2-10), the driver genes' enrichment test (by hypergeometric test) is always less than 0.05, which means that CDPath can identify statistically significant modules with known driver genes. The objective function value generally increases as the increase of $N_{m}$. This observation certifies that the additionally identified modules also embody the characteristics of cooperative driver pathways. Therefore, we can conclude that with different settings of $N_{m}$, CDPath can always find significant driver core modules.

TABLE 3

Driver genes identified by CDPath on BRCA dataset.

\begin{tabular}{l|l|l|l}
\hline$N_{m}$ & Known Drivers & Driver Enrichment & Objective Value \\
\hline 2 & 5 & $3.09 \mathrm{E}-9$ & 31.62 \\
\hline 3 & 5 & $1.12 \mathrm{E}-8$ & 50.15 \\
\hline 4 & 7 & $8.31 \mathrm{E}-12$ & 72.80 \\
\hline 5 & 7 & $9.69 \mathrm{E}-11$ & 102.78 \\
\hline 6 & 8 & $1.84 \mathrm{E}-12$ & 111.32 \\
\hline 7 & 9 & $8.30 \mathrm{E}-10$ & 116.89 \\
\hline $\mathbf{8}$ & $\mathbf{1 1}$ & $\mathbf{6 . 6 6 E}-16$ & $\mathbf{1 3 8 . 5 6}$ \\
\hline 9 & 9 & $1.98 \mathrm{E}-13$ & 145.92 \\
\hline 10 & 10 & $6.67 \mathrm{E}-16$ & 124.31 \\
\hline
\end{tabular}

$N_{m}$ : number of identifed modules. Known Drivers: the number of known driver genes in identified modules. Objective Value: objective function value of CDPath.

TABLE 4

Driver genes identified by CDPath on UCEC dataset.

\begin{tabular}{l|l|l|l}
\hline$N_{m}$ & Known Drivers & Driver Enrichment & Objective Value \\
\hline 2 & 7 & $4.97 \mathrm{E}-14$ & 60.38 \\
\hline 3 & 8 & $1.58 \mathrm{E}-14$ & 105.86 \\
\hline 4 & 8 & $3.02 \mathrm{E}-13$ & 147.82 \\
\hline 5 & 8 & $3.02 \mathrm{E}-13$ & 167.43 \\
\hline $\mathbf{6}$ & $\mathbf{1 0}$ & $\mathbf{9 . 9 9 E - 1 9}$ & $\mathbf{2 2 5 . 6 6}$ \\
\hline 7 & 10 & $1.11 \mathrm{E}-16$ & 177.92 \\
\hline 8 & 8 & $1.78 \mathrm{E}-11$ & 236.36 \\
\hline 9 & 9 & $4.22 \mathrm{E}-15$ & 193.46 \\
\hline 10 & 8 & $1.73 \mathrm{E}-12$ & 98.27 \\
\hline
\end{tabular}

After choosing a best $N_{m}$ for BRCA and UCEC, the identified driver core modules and the relationships among them are shown in Figure 2 and Figure 3 , respectively. We can see that there are a lot of ME edges within and $\mathrm{CO}$ (functional) edges between modules. This result proves that CDPath can accurately group gene sets to form cooperative driver pathways.

As Figure 2 shows, there are 8 modules, including 6 known drivers in breast cancer, they are TP53, AKT1, CDH1, PIK3CA, GATA3 and MAP3K1. CDPath can find genes with functional relations. For example, PIK3CA is a member of the PIK3C/AKT1 pathway, therefore, PIK3CA and AKT1 
are closely related. CDPath can also find genes with cooccurrence. For example, NCOR2 (nuclear co-repressor) and NCOA3 (co-activator), and the latter is a well-known cancer driver [30]. As for the genes currently not reported as cancer genes, many of them are involved with signaling. For example, MUC4 contributes to tumor progression by promoting cell survival [31]-[33]. The increased mutations of TTN in breast cancer are possible linked with APOBEC activity [24], which means that TTN mutations may be a disease trigger [34]. We also examine the associations between the modules and traditional breast cancer subtypes, as well as their associations with mutagenic processes, which are independent from the traditional subtypes. We find that the mutations in the PIK3CA/CDHA and MAP3K1/MAP2K4 are significantly enriched in the Luminal A subtype. NCOA3/TBL1XR1/NCOR2/MED23 are moderately associated with the Luminal A subtype. Strong association between both MAP3K1 and MAP2K4 genes and the Luminal A subtype is in agreement with the previous finding that mutations in MAP2K4 produce perturbations similar to MAP3K1 loss [35]. On the other hand, the mutations in the TTN/NEB/DMD module is enriched in the Her2 and Basal subtypes.

We further do Disease, GO and KEGG enrichment analysis for the whole identified genes, as Table 5 shows, they show associations with breast cancer and with several carcinogenesis related activities, such as cell death, gene expression and so on. Enriched pathways, including VEGF, EGF and ErbB, have been reported to be related with breast cancer. In summary, $96.2 \%$ of the identified genes are involved with important biological activities.

As Figure 3 shows, we identify 6 modules that include many prominent cancer drivers/associated genes in endometrial cancer, such as TP53, CCND1, KRAS, PTEN, PIK3CA, PIK3R1, RPL22, and ARID1A. We also retrieved a module related to nonsense mediated decay (SMG1, SMG7). SMG7 is also known to regulate p53 stability and function in DNA damage stress response. As Table 6 shows, the identified genes are related with endometrial cancer and with carcinogenesis related activities, including cell death, cell growth and etc. These genes are enriched with cancer related pathways, including endometrial cancer and Wnt signaling pathway. In summary, $92.6 \%$ of the identified genes are involved with important biological activities.

In order to testify the functional relations of the identified genes at the pathway level, we also do permutation tests on the identified cooperative gene modules. We iteratively and randomly select the same number of genes of identified cooperative driver pathways from all the candidate genes 100 times, and do Disease, KEGG and GO functional enrichment analysis on these random gene sets. For BRCA dataset, only $0,5(5 \%)$ and $18(18 \%)$ of the random gene sets are enriched in Disease, KEGG and GO functional enrichment analysis, respectively. For UCEC dataset, only $0,2(2 \%)$ and $22(22 \%)$ of the random gene sets are enriched in Disease, GO and KEGG functional enrichment analysis, respectively. It is obviously that the probability of GO enrichment is much higher than KEGG and Disease. That is because most genes are related with some important biological activities to some extent. However, when analyzing these random gene sets at the pathway level, they do not show signifi- cant Disease and KEGG functional enrichment. Therefore, the probability of identifying such significant gene sets by chance is very low. This permutation test confirms that our method can effectively identify gene sets that compose cooperative driver pathways rather than single driver genes.

Based on the above analysis on the identified modules, we can conclude that CDPath can effectively identify modules with close associations with the target cancer and important biological activities involved with cancer development.



Fig. 2. Driver core modules identified by CDPath on BRCA. The edges have same meanings as Figure 1 The known driver genes are colored with deep red.



Fig. 3. Driver core modules identified by CDPath on UCEC. The edges have same meanings as Figure 1 . The known driver genes are colored with deep red.

\subsubsection{Identified cooperative driver pathways analysis}

By using MCL on PaPaI network, we get 37 pathway clusters, after combining these clusters with pathway modules, we finally obtain 28 and 30 cooperative driver pathway sets on BRCA and UCEC, respectively. Table 8 lists several representative ones, which are related with the target disease and supported by biomedical literature.

For BRCA dataset, we find EGF receptor (ErbB1) signaling pathway, IGF1 pathway, ErbB1 downstream signaling 
TABLE 5

Functional analysis of identified modules on BRCA

\begin{tabular}{llll}
\hline Category & Terms & P-value & Genes \\
\hline Disease & Breast cancer & $2.3 \mathrm{E}-2$ & AKT1, PIK3CA \\
\hline GO & positive regulation of transcription & $4.59 \mathrm{E}-4$ & TBL1XR1, NCOA3, MAP3K1, TP53, ARID1A, RUNX1, CBFB \\
\hline GO & positive regulation of gene expression & $5.38 \mathrm{E}-4$ & TBL1XR1, NCOA3, MAP3K1, TP53, ARID1A, RUNX1, CBFB \\
\hline GO & regulation of cell death & $3.10 \mathrm{e}-3$ & RPL18, EEF1B2, RPL27A, RPLP2, RPL36, RPL38, RPL39, RPS28 \\
\hline KEGG & Pathways in cancer & $1.01 \mathrm{E}-5$ & AKT1, LAMA2, PLCG1, TP53, PIK3CA, CDH1, RUNX1, PTEN \\
\hline KEGG & ErbB signaling pathway & $1.53 \mathrm{E}-3$ & AKT1, PLCG1, MAP2K4, PIK3CA \\
\hline KEGG & EGF Signaling Pathway & $2.07 \mathrm{E}-3$ & PLCG1, MAP3K1, MAP2K4, PIK3CA \\
\hline KEGG & VEGF signaling pathway & $1.74 \mathrm{E}-2$ & AKT1, PLCG1, PIK3CA \\
\hline
\end{tabular}

TABLE 6

Functional analysis of identified modules on UCEC

\begin{tabular}{llll}
\hline Category & Terms & P-value & Genes \\
\hline Disease & endometrial cancer & $3.42 \mathrm{E}-4$ & CCND1, KRAS, TP53, PTEN \\
\hline GO & cell death & $9.14 \mathrm{E}-3$ & EP300, KRAS, TP53, SPTBN2, PTEN, CTNNB1 \\
\hline GO & regulation of cell growth & $5.29 \mathrm{E}-3$ & PPP2R1A, EP300, TP53, MUC12 \\
\hline GO & T cell differentiation & $6.35 \mathrm{e}-3$ & RPL22, TP53, CTNNB1 \\
\hline KEGG & Endometrial cancer & $2.40 \mathrm{E}-9$ & CCND1, KRAS, TP53, PIK3CA, PTEN, PIK3R1, CTNNB1 \\
\hline KEGG & Pathways in cancer & $5.88 \mathrm{E}-7$ & LAMA2, CCND1, EP300, KRAS, TP53, PIK3CA, PTEN, PIK3R1, CTNNB1 \\
\hline KEGG & Wnt signaling pathway & $5.93 \mathrm{E}-4$ & PPP2R1A, CCND1, EP300, TP53, CTNNB1 \\
\hline
\end{tabular}

and their cooperative pathways. It is recognized that the occurrence, development, metastasis and drug resistance of breast cancer are closely related to intracellular signaling pathways, including estrogen receptor (ER) signaling pathway and insulin-like growth factor receptor (IGFR) signaling pathway and epidermal growth factor receptor (EGFR) signaling pathway, all of which are particularly important [36]. For NF-kappaB pathway, its inhibitors preferentially inhibit breast cancer stem-like cells [37]. As for PI3K pathway, it is altered in a high proportion of breast cancers and may contribute to therapeutic resistance [38]. In addition, Notch signaling pathway and p53 pathway are well-known cancer pathways |39|.

For UCEC dataset, we also identify p53 pathway, the van-cancer pathway, which can lead to endometrial cancer when being perturbed. As for PI3K pathway, the overexpressed HOTTIP promotes the development of endometrial cancer via activating PI3K pathway [40|. By disturbing the AKT/mTOR and MAPK signaling pathways, novasoy and genistein can inhibit endometrial cancer cell proliferation [41].

Based on the above analysis, we can state that CDPath can effectively identify the target cancer related cooperative pathways, and the cooperation between them can be further investigated by wet experiments.

\subsection{Comparison with other methods}

To further confirm the effectiveness of CDPath, we compare it against four representative and competitive methods, including individual driver pathway identification methods: Dendrix [12] and BLP [13]; and multiple driver pathway identification methods: CoMDP $|20|$ and Multi-Dendrix [21|. These four comparing approaches were introduced in the Introduction section. Since these comparing methods do not find exact pathways, we use the known gene-pathway associations to find the corresponding pathways. More specifically, we collect all the related pathways for each gene of the driver gene set, and identify pathways commonly related to at least 2 genes as (individual/cooperative) driver pathways.
We firstly compare our method with these methods at gene level. As Table 7 shows, in both BRCA and UCEC datasets, our method outperforms all the comparing methods on the number of known driver genes and driver genes enrichment analysis (which can avoid the bias of statistical significance caused by different number of identified genes). Moreover, all the driver genes (except MLL3) identified by other methods are all identified by CDPath. Therefore, CDPath can more accurately identify more significant genes than these comparing approaches.

Two factors account for the superiority of CDPath to these comparing methods, which are extended from Dendrix and can only identify one or two pathways with high coverage and mutual exclusivity on the mutation data. In contrast, CDPath can flexibly identify more significant gene modules by importing both mutation data and PPI network via ILP. In addition, CDPath is more flexible than these methods, it does not need to specify the number of identified genes beforehand, whereas these methods need. CDPath only needs to specify the number of modules, which can be easily selected by considering the value of module benefit (see Figure S2 of the Supplementary file) and the number of driver genes.

We further compare CDPath with other methods at the pathway level. As Table 8 shows, pathways identified by CDPath cover more breast cancer related pathways (supported by biomedical literature) and have overlap with other methods, which are highlighted in bold font. For example, in BRCA dataset, CDPath identifies the RAC1 signaling pathway, which is also contained by Dendrix. RAC1 signaling pathway has been reported to be related with ovarian cancer, but not yet with the breast cancer. Therefore, RAC1 signaling pathway may be a high potential driver pathway of breast cancer. Furthermore, compared to Dendrix, CDPath also identifies its cooperative pathway Class I PI3K signaling events, which have been reported to be related with breast cancer. Another example is that in UCEC dataset, CDPath also identifies the only driver pathway p53 pathway identified by BLP. Therefore, CDPath can not only identify more driver pathways than individ- 
TABLE 7

Known driver gene sets of BRCA and of UCEC identified by CDPath and other comparing methods.

\begin{tabular}{|c|c|c|c|c|}
\hline Method & Driver Genes of BRCA & Enrichment of BRCA & Driver Genes of UCEC & Enrichment of UCEC \\
\hline CDPath & $\begin{array}{l}\text { PIK3CA, CDH1,RUNX1, } \\
\text { GATA3,ARID1A,ATM,NCOA3 } \\
\text { TP53,MAP3K1,PTEN,AKT1 }\end{array}$ & $6.66 \mathrm{E}-16$ & $\begin{array}{l}\text { ARID1A,TP53,FBXW7, } \\
\text { KRAS,PTEN,PIK3CA, } \\
\text { PPP2R1A,CTNNB1,PIK3R1,EP300 }\end{array}$ & 9.99E-19 \\
\hline Dendrix & CDH1,TP53 & $2.56 \mathrm{E}-03$ & PIK3CA,PIK3R1 & $1.83 \mathrm{E}-03$ \\
\hline BLP & TP53, PIK3CA, GATA3 & $5.19 \mathrm{E}-05$ & TP53, PTEN & $1.83 \mathrm{E}-03$ \\
\hline Multi-Dendrix & CDH1, TP53, GATA3, PIK3CA & $6.85 \mathrm{E}-07$ & PIK3CA, PIK3R1 & $1.83 \mathrm{E}-03$ \\
\hline CoMDP & $\begin{array}{l}\text { MAP3K1, TP53, CDH1, } \\
\text { GATA3, MLL3, PIK3CA }\end{array}$ & 3.81E-11 & $\begin{array}{l}\text { PIK3R1, FBXW7, PIK3CA, } \\
\text { PPP2R1A, TP53, CTNNB1, PTEN }\end{array}$ & 4.97E-14 \\
\hline
\end{tabular}

TABLE 8

Driver pathways of BRCA and UCEC identified by CDPath and other comparing methods.

\begin{tabular}{|c|c|c|}
\hline Method & Driver Pathways of BRCA & Driver Pathways of UCEC \\
\hline \multirow{8}{*}{ CDPath } & $\begin{array}{l}\text { p57(NTR)-mediated signaling, } 42 \\
\text { VEGFR3 signaling in lymphatic endothelium; } 36\end{array}$ & \multirow[b]{2}{*}{$\begin{array}{l}\text { Class I PI3K signaling events, } 40] \\
\text { Cabonical Wnt signaling pathway; } 43]\end{array}$} \\
\hline & $\begin{array}{l}\text { Class I PI3K signaling events mediated by Akt, } 38 \text {. } \\
\text { Atypical NF-kappaB pathway; [37] }\end{array}$ & \\
\hline & ErbB1 downstream signaling,ErbB2ErbB3 signaling events; & \multirow{2}{*}{$\begin{array}{l}\text { mTOR signaling pathway, } \\
\text { Notch signaling pathway; }\end{array}$} \\
\hline & EGF receptor (ErbB1) signaling pathway, Notch signaling pathway; 36 & \\
\hline & IGF1 pathway, Integrins in angiogenesis; 45 & \multirow{2}{*}{$\begin{array}{l}\text { II2 signaling events mediated by PI3K, } 40 \\
\text { mTOR signaling pathway; [41] }\end{array}$} \\
\hline & p53 pathway, FoxO family signaling; 39 & \\
\hline & RAC1 signaling pathway, Class I PI3K signaling events; 38 & \multirow{2}{*}{$\begin{array}{l}\text { p53 pathway, [46] } \\
\text { IL2 signaling events mediated by STAT5; }\end{array}$} \\
\hline & Internalization of ErbB1, 44 & \\
\hline \multirow[b]{2}{*}{ Dendrix } & RAC1 signaling pathway, & \multirow[b]{2}{*}{$\begin{array}{l}\text { Class I PI3K signaling events, } 40 \\
\text { p53 pathway } 46\end{array}$} \\
\hline & $\begin{array}{l}\text { CDC42 signaling events, } \\
\text { Signaling events mediated by Hepatocyte Growth Factor Receptor (c-Met) }\end{array}$ & \\
\hline BLP & $\begin{array}{l}\text { p53 pathway, } 39 \\
\text { p75(NTR)-mediated signaling, } 42 \\
\text { Glucocorticoid receptor regulatory network, } \\
\text { Validated targets of C-MYC transcriptional activation, } 47 \\
\text { Integrins in angiogenesis }\end{array}$ & p53 pathway 46 \\
\hline Multi-Dendrix & $\begin{array}{l}\text { RAC1 signaling pathway, } \\
\text { Class I PI3K signaling events; } 38\end{array}$ & $\begin{array}{l}\text { Class I PI3K signaling events, } 40 \\
\text { IL2 signaling events mediated by STAT5 }\end{array}$ \\
\hline \multirow{3}{*}{ CoMDP } & p53 pathway, ErbB1 downstream signaling; 39,44 & \multirow{3}{*}{$\begin{array}{l}\text { Class I PI3K signaling events, } 40] \\
\text { IL2 signaling events mediated by PI3K; } 40\end{array}$} \\
\hline & p53 pathway, p75(NTR)-mediated signaling; 4 & \\
\hline & $\begin{array}{l}\text { p53 pathway, Signaling events mediated } \\
\text { by Hepatocyte Growth Factor Receptor (c-Met); }\end{array}$ & \\
\hline \multirow{2}{*}{ CDPath(nMCL) } & $\begin{array}{l}\text { ErbB1 downstream signaling, } \\
\text { Class I PI3K signaling events mediated by Akt; } 38\end{array}$ & \multirow{2}{*}{$\begin{array}{l}\text { IL2 signaling events mediated by STAT5, } \\
\text { AP-1 transcription factor network; } \\
\text { Internalization of ErbB1; } \\
\text { Class I PI3K signaling events } 40\end{array}$} \\
\hline & $\begin{array}{l}\text { VEGFR1 specific signals, } 36 \\
\text { ErbB2ErbB3 signaling events; } 44\end{array}$ & \\
\hline
\end{tabular}

CDPath(nMCL): CDPath without MCL on PaPaI network. Overlapped driver pathways identified by CDPath and comparing methods are highlighted in boldface font.

ual methods but also uncover more potential cooperations between pathways. We can easily find that these individual pathway identification methods focus on identifying only a driver gene set, while CDPath can uncover the cooperations between pathways by importing pathway interaction network.

As to the multiple pathway methods, Multi-Dendrix and CoMDP, the results of CDPath also have overlaps with them. Specifically, the same pathway set RAC1 signaling pathway and Class I PI3K signaling events with Multi-Dendrix on the BRCA dataset, and CDPath can discover more cooperations. In addition, because of the main effect of gene TP53, in BRCA (UCEC) dataset, pathway sets identified by CoMDP (Multi-Dendrix) all contain p53 pathway, thus the diversity of cooperation pathways identified by CoMDP drops sharply. In contrast, CDPath holds a good diversity, that is because CDPath restricts each gene belonging to one module and thus avoids the main effect without removing any genes. CDPath outperforms these multiple pathway methods, since it not only accounts for the properties of cooperative driver pathways as these comparing methods do, but also effectively uncovers cooperations among significant pathway modules by MCL on the pathway interactive network.
In addition, we also test CDPath without the process of MCL on PaPaI, and identify several pathway sets using known gene-pathway associations. As mentioned before, because of the incomplete gene-pathway association information, there is no corresponding driver pathways for 4/8 (50\%) and 3/6 (50\%) modules of BRCA and UCEC, respectively. These results means that half driver pathways are lost. Table 8 shows several driver pathway sets with supportive biomedical literature. We can see that, if MCL is not applied, the number of identified driver pathway sets obtained by CDPath(nMCL) drops sharply, and the driver pathways identified by CDPath is two times more than CDPath(nMCL). Therefore, we can state that MCL on $\mathrm{PaPaI}$ network helps identifying exact cooperative driver pathways.

\section{CONCLUSION}

Identifying cooperative driver pathways of complex disease is crucial for precise treatment. In this paper, we study how to discover cooperative driver pathways and introduce a computational approach. We firstly use Integer linear programming to identify cooperative driver core modules by simultaneously accounting for four characteristics of 
cooperative driver pathways: coverage, mutual exclusivity, co-occurrences and functional cooperation. Based on simulation experiments and driver genes enrichment tests, these identified modules show statistical and biological significance. To facilitate exact cooperative driver pathways identification, we then apply Markov clustering on the pathway interaction network and obtain pathway clusters with functional cooperation relations. Finally, we take pathways that belong to different modules but in the same pathway clusters as cooperative driver pathways. These identified cooperative pathways are mostly supported by biomedical literature. Compared with other pathway identification approaches, CDPath can uncover the underlying relationships between genes, gene groups and pathways that are not accessible by individual driver pathway identification methods, and it can discover more known driver pathways than existing cooperative driver pathway discovery methods. We believe that biological experiments can be done based on our new findings of genes and pathways, especially some genes we listed, such as SMG7 for endometrial cancer. It may be important to discover the transcriptions of these genes and how they interact with other genes in patients.

In the future work, we will try to enhance the effectiveness of identifying core modules by incorporating more characteristics of cooperative driver pathways, and to use multiple types of data and interactions among genes and pathways to guide the cooperative driver modules identification.

\section{ACKNOWLEDGEMENTS}

This work is supported by Natural Science Foundation of China (61873214, 61872300, 61871020, 61571163 and 61532014), Fundamental Research Funds for the Central Universities (XDJK2019B024), the National Key Research and Development Plan Task of China (Grant No.2016YFC0901902), Natural Science Foundation of CQ CSTC (cstc2018jcyjAX0228), and the Fundamental Research Funds for the Central Universities, NWSUAF, China (2452015060).

\section{RefERENCES}

[1] C. G. A. R. Network, "Integrated genomic analyses of ovarian carcinoma," Nature, vol. 474, no. 7353, pp. 609-615, 2011.

[2] I. C. G. Consortium, "International network of cancer genome projects," Nature, vol. 464, no. 7291, pp. 993-998, 2010.

[3] R. Beroukhim, G. Getz, L. Nghiemphu, J. Barretina, T. Hsueh, D. Linhart, I. Vivanco, J. C. Lee, J. H. Huang, and S. Alexander, "Assessing the significance of chromosomal aberrations in cancer: Methodology and application to glioma," Proceedings of the $\mathrm{Na}$ tional Academy of Sciences of the United States of America, vol. 104, no. 50, pp. 20007-20012, 2007.

[4] L. Ding, G. Getz, D. A. Wheeler, E. R. Mardis, M. D. Mclellan, K. Cibulskis, C. Sougnez, H. Greulich, D. M. Muzny, and M. B. Morgan, "Somatic mutations affect key pathways in lung adenocarcinoma," Nature, vol. 455, no. 7216, pp. 1069-1075, 2008.

[5] M. Vazquez, V. D. L. Torre, and A. Valencia, "Chapter 14: Cancer genome analysis," PLoS Computational Biology, vol. 8, no. 12, p. e1002824, 2012

[6] S. Jones, X. Zhang, D. W. Parsons, C. H. Lin, R. J. Leary, P. Angenendt, P. Mankoo, H. Carter, H. Kamiyama, and A. Jimeno, "Core signaling pathways in human pancreatic cancers revealed by global genomic analyses," Science, vol. 321, pp. 1801-1806, 2008.

[7] J. Zhang and S. Zhang, "The discovery of mutated driver pathways in cancer: Models and algorithms," IEEE/ACM Trans. Comput. Biol. Bioinformatics, vol. 15, no. 3, pp. 988-998, 2018.
[8] F. Vandin, E. Upfal, and B. J. Raphael, "Algorithms for detecting significantly mutated pathways in cancer," Journal of Computational Biology, vol. 18, no. 3, pp. 507-522, 2011.

[9] B. Vogelstein and K. W. Kinzler, "Cancer genes and the pathways they control," Nature Medicine, vol. 10, no. 8, pp. 789-799, 2004.

[10] J. Zhang, S. Zhang, Y. Wang, and X. S. Zhang, "Identification of mutated core cancer modules by integrating somatic mutation, copy number variation, and gene expression data," BMC Systems Biology, vol. 7, no. 2, p. S4, 2013.

[11] G. Ciriello, E. Cerami, C. Sander, and N. Schultz, "Mutual exclusivity analysis identifies oncogenic network modules," Genome Research, vol. 22, no. 2, pp. 398-406, 2012.

[12] F. Vandin, E. Upfal, and B. J. Raphael, "De novo discovery of mutated driver pathways in cancer," Genome Research, vol. 22, no. 2, pp. 375-385, 2012.

[13] J. Zhao, S. Zhang, L. Y. Wu, and X. S. Zhang, "Efficient methods for identifying mutated driver pathways in cancer," Bioinformatics, vol. 28, no. 22, pp. 2940-2947, 2012.

[14] J. Zhang and S. Zhang, "Discovery of cancer common and specific driver gene sets," Nucleic Acids Research, vol. 45, no. 10, p. e86, 2017.

[15] D. Hanahan and R. A. Weinberg, "Hallmarks of cancer: the next generation," Cell, vol. 144, no. 5, pp. 646-674, 2011.

[16] C. H. Yeang, F. Mccormick, and A. Levine, "Combinatorial patterns of somatic gene mutations in cancer," The FASEB Journal, vol. 22, no. 8, pp. 2605-2622, 2008.

[17] Q. Cui, M. Yun, M. Jaramillo, H. Bari, A. Awan, Y. Song, S. Zhang, L. Liu, L. Meng, and M. O'Connormccourt, "A map of human cancer signaling," Molecular Systems Biology, vol. 3, p. 152, 2007.

[18] Y. Gu, W. Zhao, J. Xia, Y. Zhang, R. Wu, C. Wang, and Z. Guo, "Analysis of pathway mutation profiles highlights collaboration between cancer-associated superpathways," Human Mutation, vol. 32, no. 9, pp. 1028-1035, 2011.

[19] Y. Gu, H. Wang, Y. Qin, Y. Zhang, W. Zhao, L. Qi, Y. Zhang, C. Wang, and Z. Guo, "Network analysis of genomic alteration profiles reveals co-altered functional modules and driver genes for glioblastoma," Molecular Biosystems, vol. 9, no. 3, pp. 467-477, 2013.

[20] J. Zhang, L. Y. Wu, X. S. Zhang, and S. Zhang, "Discovery of cooccurring driver pathways in cancer," BMC Bioinformatics, vol. 15, no. 1, pp. 271-285, 2014.

[21] M. D. Leiserson, D. Blokh, R. Sharan, and B. J. Raphael, "Simultaneous identification of multiple driver pathways in cancer," PLOS Computational Biology, vol. 9, no. 5, p. e1003054, 2013.

[22] P. Dao, Y. A. Kim, D. Wojtowicz, S. Madan, R. Sharan, and T. M. Przytycka, "Bewith: A between-within method to discover relationships between cancer modules via integrated analysis of mutual exclusivity, co-occurrence and functional interactions," PLoS Computational Biology, vol. 13, no. 10, p. e1005695, 2017.

[23] S. Van Dongen, "Graph clustering by flow simulation," PhD Thesis University of Utrecht, 2000.

[24] Y. A. Kim, S. Madan, and T. M. Przytycka, "Wesme: Uncovering mutual exclusivity of cancer drivers and beyond," Bioinformatics, vol. 33, no. 6, pp. 814-821, 2016.

[25] S. Brohee and J. V. Helden, "Evaluation of clustering algorithms for protein-protein interaction networks," BMC Bioinformatics, vol. 7 , no. 1 , p. 488,2006

[26] V. James and S. J. Wodak, "Markov clustering versus affinity propagation for the partitioning of protein interaction graphs," BMC Bioinformatics, vol. 10, no. 1, p. 99, 2009.

[27] Y. K. Shih and S. Parthasarathy, "Identifying functional modules in interaction networks through overlapping markov clustering," Bioinformatics, vol. 28, no. 18, pp. i473-i479, 2012.

[28] C. F. Schaefer, K. Anthony, S. Krupa, J. Buchoff, M. Day, T. Hannay, and K. H. Buetow, "Pid: the pathway interaction database," Nucleic Acids Research, vol. 37, no. S1, pp. 674-D679, 2009.

[29] C. L. M. M. D. T. H. T. W. R. . S. M. R. . Futreal, P. A., "A census of human cancer genes," Nature Reviews Cancer, vol. 4, no. 3, p. 177, 2004.

[30] B. Vogelstein, N. Papadopoulos, V. E. Velculescu, S. Zhou, D. L. Jr, and K. W. Kinzler, "Cancer genome landscapes," Science, vol. 339, no. 6127, pp. 1546-1558, 2013.

[31] S. Bafna, S. Kaur, and S. K. Batra, "Membrane-bound mucins: the mechanistic basis for alterations in the growth and survival of cancer cells," Oncogene, vol. 29, no. 20, pp. 2893-2904, 2010.

[32] D. W. Kufe, "Mucins in cancer: function, prognosis and therapy." Nature Reviews Cancer, vol. 9, no. 12, pp. 874-885, 2009. 
[33] M. A. Hollingsworth and B. J. Swanson, "Mucins in cancer: protection and control of the cell surface," Nature Reviews Cancer, vol. 4, no. 1, pp. 45-60, 2004.

[34] M. Hofree, J. P. Shen, H. Carter, A. Gross, and T. Ideker, “Networkbased stratification of tumor mutations," Nature Methods, vol. 10, no. 11, pp. 1108-1115, 2013.

[35] M. J. Ellis, L. Ding, D. Shen, J. Luo, V. J. Suman, J. W. Wallis, B. A. V. Tine, J. Hoog, R. J. Goiffon, and T. C. Goldstein, "Whole genome analysis informs breast cancer response to aromatase inhibition," Nature, vol. 486, no. 7403, pp. 353-360, 2012.

[36] M. Elbaz, M. W. Nasser, J. Ravi, N. A. Wani, D. K. Ahirwar, H. Zhao, S. Oghumu, A. R. Satoskar, K. Shilo, and W. E. C. Iii, "Modulation of the tumor microenvironment and inhibition of egf/egfr pathway: Novel anti-tumor mechanisms of cannabidiol in breast cancer," Molecular Oncology, vol. 9, no. 4, pp. 906-919, 2015.

[37] J. Zhou, H. Zhang, P. Gu, J. Bai, J. B. Margolick, and Y. Zhang, "Nf-kappab pathway inhibitors preferentially inhibit breast cancer stem-like cells," Breast Cancer Research and Treatment, vol. 111, no. 3, pp. 419-427, 2008.

[38] E. Lpezknowles, S. A. O'Toole, C. M. Mcneil, E. K. Millar, M. R. Qiu, P. Crea, R. J. Daly, E. A. Musgrove, and R. L. Sutherland, "Pi3k pathway activation in breast cancer is associated with the basal-like phenotype and cancer-specific mortality." International Journal of Cancer, vol. 126, no. 5, p. 1121C1131, 2010.

[39] M. Gasco, S. Shami, and T. Crook, "The p53 pathway in breast cancer," Breast Cancer Research, vol. 4, no. 2, pp. 70-76, 2002.

[40] Z. Q. Z. C. L. Q. Guan, Q. and Q. L. Ren, "Hottip regulates progression of endometrial cancer via activating pi3k/akt pathway," European Review for Medical and Pharmacological Sciences, vol. 22, no. 12 , pp. 3727-3733, 2018

[41] K. M. Malloy, J. Wang, L. H. Clark, Z. Fang, W. Sun, Y. Yin, W. Kong, C. Zhou, and V. L. Baejump, "Novasoy and genistein inhibit endometrial cancer cell proliferation through disruption of the akt/mtor and mapk signaling pathways." American Journal of Translational Research, vol. 10, no. 3, pp. 784-795, 2018.

[42] J. P. Wilmet, C. Tastet, E. Desruelles, N. Ziental-Gelus, V. Blanckaert, H. Hondermarck, and B. X. Le, "Proteome changes induced by overexpression of the p75 neurotrophin receptor (p75ntr) in breast cancer cells," International Journal of Developmental Biology, vol. 55, no. 7-8-9, pp. 801-809, 2011.

[43] M. Van der Zee, Y. Jia, Y. Wang, C. Heijmans-Antonissen, P. C. Ewing, P. Franken, F. J. DeMayo, J. P. Lydon, C. W. Burger, R. Fodde et al., "Alterations in wnt- $\beta$-catenin and pten signalling play distinct roles in endometrial cancer initiation and progression," Journal of Pathology, vol. 230, no. 1, pp. 48-58, 2013.

[44] S. P. Bagaria, P. S. Ray, M. S. Sim, X. Ye, J. M. Shamonki, X. Cui, and A. E. Giuliano, "Personalizing breast cancer staging by the inclusion of er, pr, and her2," JAMA Surgery, vol. 149, no. 2, pp. 125-129, 2014.

[45] C. Y. Sun, X.-H. Li, W. Lu, X.-F. Hu, J.-X. Dai, and L. Yuan, "Igf-1 from adipose-derived mesenchymal stem cells promotes radioresistance of breast cancer cells," Asian Pacific Journal of Cancer Prevention, vol. 15, no. 23, pp. 10115-10119, 2014.

[46] M. E. Sherman, M. E. Bur, and R. J. Kurman, "p53 in endometrial cancer and its putative precursors: evidence for diverse pathways of tumorigenesis," Human Pathology, vol. 26, no. 11, pp. 1268-1274, 1995.

[47] Z. Li, Q. Meng, Q. Yu, Z. Zhou, and L. Li, "Evaluation of c-myc and ccne2 amplification in breast cancer with quantitative multi-gene fluorescence in-situ hybridization," Chinese Journal of Pathology, vol. 43, no. 7, pp. 455-458, 2014.

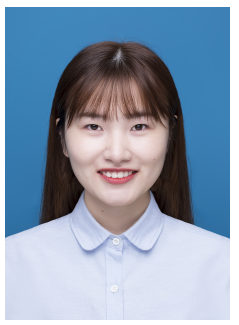

Ziying Yang is an MPhil student at the College of Computer and Information Sciences, Southwest University, Chongqing, China. She received B.Sc. degree in Digital Media Technology from Yunnan University, Kunming, China. Her research interests include data mining and bioinformatics.

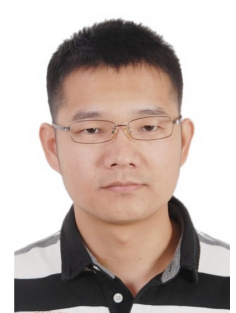

Guoxian $\mathrm{Yu}$ is a Professor at the College of Computer and Information Science, Southwest University, Chongqing, China. He received the Ph.D. in Computer Applied Technology from South China University of Technology, Guangzhou, China in 2013. His current research interests include data mining and bioinformatics. He is a recipient of Best Poster Award of SDM2012 Doctral Forum and Best Student Paper Award of IEEE International Conference on Machine Learning and Cybernetics, 2011. He has served as PC member for KDD, IJCAI, AAAI, ICDM, SDM, ISBRA and reviewer for IEEE Trans. Journals.

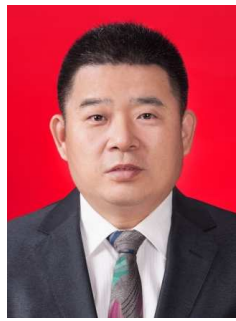

Maozu Guo is a professor at the College of Electrical and Information Engineering, Beijing University of Civil Engineering and Architecture, Beijing, China. He received the Ph.D. degree in Computer Science and Technology from Harbin Institute of Technology. His research interests include bioinformatics, machine learning, and data mining.

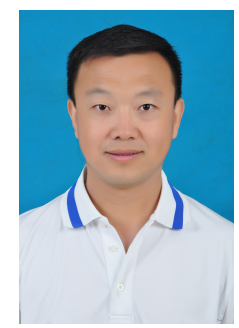

Jiantao $\mathrm{Yu}$ is a lecturer at the College of College of Information Engineering, North-West A\&F University, Shaanxi, China. He received the Ph.D. degree in Artificial Intelligence from Harbin Institute of Technology. His research interests include machine learning and bioinformatics.



Xiangliang Zhang is an Associate Professor and directs the Machine Intelligence and Knowledge Engineering (MINE) Laboratory in King Abdullah University of Science and Technology (KAUST). She earned her PhD degree in Computer Science with great honors from INRIAUniversity Paris-Sud 11, France, in 2010. Her main research interests and experiences are in diverse areas of machine learning and data mining.

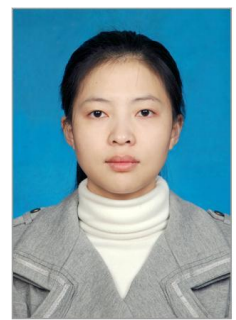

Jun Wang is an Associate Professor in the College of Computer and Information Science, Southwest University, Chongqing, China. She received B.Sc. degree in Computer Science, M.Eng. degree in Computer Science and Ph.D. in Artificial Intelligence from Harbin Institute of Technology, Harbin, China in 2004, 2006 and 2010 , respectively. Her current research interests include machine learning, data mining and their applications in bioinformatics. 